(C) The Author(s), 2020. Published by Cambridge University Press on behalf of The Nutrition Society. This is an Open Access article, distributed under the terms of the Creative Commons Attribution licence (http://creativecommons.org/licenses/by/4.0/), which permits unrestricted re-use, distribution, and reproduction in any medium, provided the original work is properly cited.

\title{
Intake of Calanus finmarchicus oil for 12 weeks improves omega-3 index in healthy older subjects engaging in an exercise programme
}

\author{
Paulina Wasserfurth ${ }^{1}$, Josefine Nebl ${ }^{1}$, Tim Konstantin Boßlau ${ }^{2}$, Karsten $\mathrm{Krüger}^{2}$, Andreas Hahn ${ }^{1}$ \\ and Jan Philipp Schuchardt ${ }^{1 *}$ \\ ${ }^{1}$ Faculty of Natural Sciences, Institute of Food Science and Human Nutrition, Leibniz University Hannover, 30167 Hannover, \\ Germany \\ ${ }^{2}$ Department of Exercise Physiology and Sports Therapy, Institute of Sports Science, Justus-Liebig-University Giessen, 35394 \\ Giessen, Germany
}

(Submitted 14 May 2020 - Final revision received 10 July 2020 - Accepted 18 July 2020 - First published online 23 July 2020)

Abstract

The $n$-3 PUFA, EPA and DHA, play an important role in human health. As the intake of EPA and DHA from the diet is often inadequate, supplementation of those fatty acids is recommended. A novel source of $n$ - 3 PUFA is Calanus finmarchicus oil (CO) which contains fatty acids mainly bound in wax esters. To date, no data are available on the effects of long-term intake of this marine oil on $n$-3 PUFA blood levels. Therefore, the aim of this study was to evaluate the effect of CO on the $n-3$ PUFA blood levels using the omega- 3 index (O3I). The data originate from a larger randomised controlled trial. For this analysis, samples from seventy-two participants (59.2 (sD 6.2) years, BMI $27.7(\mathrm{sD} 5.28) \mathrm{kg} / \mathrm{m}^{2}$ ) were analysed. Of those, thirty-six performed $2 \times /$ week exercise and received $2 \mathrm{~g}$ of CO, which provided $124 \mathrm{mg}$ stearidonic acid (SDA), $109 \mathrm{mg}$ EPA and $87 \mathrm{mg}$ DHA daily (EXCO group), while the other group performed exercise only (EX group) and served as a control for this analysis. The O3I increased from 6.07 (SD 1.29) \% at baseline to 7.37 (SD 1.10) \% after 12 weeks within the EXCO group $(P<0.001)$, while there were no significant changes in the EX group (6.01 (sD 1.26)-6.15 (sD 1.32) \%, $P=0.238$ ). These data provide first evidence that wax ester-bound $n-3$ PUFA from CO can significantly increase the O3I despite relatively low EPA + DHA amounts. Further, the effects of exercise could be excluded.

Key words: PUFA: Marine oil: Wax esters: EPA: DHA

n-3 PUFA are highly associated with human health, playing an important role in cardiovascular health, brain development and cognitive function as well as inflammation and inflammatory diseases $^{(1,2)}$.

Of all $n$-3 PUFA, most of those health benefits are attributed to the $20: 5 n-3$ fatty acid EPA and the $22: 6 n-3$ fatty acid DHA. Regarding their importance in human health and an unfavourable shift in the dietary fatty acid intake in favour of $n-6$ fatty acids, daily intake of at least $0.5 \mathrm{~g}$ of EPA and DHA is recommended by the International Society for the Study of Fatty Acids and Lipids (ISSFAL $)^{(3)}$. The primary dietary source for EPA and DHA is coldwater fish such as tuna, salmon, herring or mackerel; however, intake of these types of fish is often low ${ }^{(4)}$. In Germany, this is also reflected by low median dietary intakes of $65-78 \mathrm{mg}$ EPA and $107-135 \mathrm{mg} \mathrm{DHA}^{(5)}$. Therefore, supplementation of $n-3$ PUFA may be beneficial. Good $n-3$ PUFA sources that are commonly used for supplementation are marine oils, with fish oil being the most frequently used ${ }^{(6)}$. A novel $n$ - 3 PUFA source is the marine oil obtained from Calanus finmarchicus, a copepod found in the northern Atlantic sea. Unlike fish oil, where $n-3$ PUFA are bound in the form of TAG, $>80 \%$ of the fatty acids in Calanus finmarchicus oil (CO) are bound in the form of wax esters ${ }^{(7)}$. In any case, when compared with refined fish oil which commonly contains $300 \mathrm{mg}$ EPA + DHA per $\mathrm{g}^{(8)}$, the amount of EPA and DHA in CO is relatively low (about $100 \mathrm{mg}$ EPA + DHA per g). A single-dose study has already shown that EPA and DHA from CO are equally bioavailable as from fish oil ${ }^{(9)}$. However, the long-term intake of $n$-3 PUFA from $\mathrm{CO}$ has not yet been studied. The omega-3 index (O3I) (relative content of EPA + DHA in erythrocytes) has been shown to be a good and reliable indicator in evaluating the fatty acid supply over a longer period of time ${ }^{(10,11)}$.

In addition to being an indicator for the long-term fatty acid supply, an $\mathrm{O} 3 \mathrm{I}$ of $>8 \%$ was also linked to a lower risk for

Abbreviations: CO, Calanus finmarchicus oil; O3I, omega-3 index.

* Corresponding author: Jan Philipp Schuchardt, fax +49 511762 5729, email schuchardt@nutrition.uni-hannover.de 
cardiovascular events $^{(10,12)}$. This is of particular interest for all age groups engaging in exercise, as exercise increases the demands of the cardiovascular system. Although it was already demonstrated that athletes show an insufficient supply with $n-3$ PUFA $^{(13,14)}$, no data about the effects of exercise on the O3I have been described to date.

Hence, the objective of the present study was to investigate the effect of CO providing physiological EPA + DHA doses on the $\mathrm{O} 3 \mathrm{I}$ in a study collective of healthy elderly subjects who participated in an exercise programme. To account for potential effects of the exercise programme on the O3I, the group performing exercise only served as a control.

\section{Materials and methods}

\section{Study design and participants}

The present work is based on a single-centre, randomised controlled trial in parallel-group design which was conducted at the Institute of Food Science and Human Nutrition, Leibniz University Hannover, Germany. In brief, the study consisted of a screening and 12-week intervention phase with two examination days; one at the beginning $\left(t_{0}\right)$ and one at the end of the 12-week intervention $\left(t_{12}\right)$.

Participants for this study were recruited via advertisements in local newspapers and public notice boards from the general population in Hannover, Germany, between August 2018 and March 2019. The trial ended when the required sample size was achieved. The main inclusion criteria for participation were age $\geq 50$ and $\leq 70$ years, no exercise training aside the daily activities for at least 2 years, a stable body weight $( \pm 5 \mathrm{~kg})$ for at least 6 months, being able to physically perform the exercise intervention (exercise capacity) and following an omnivorous diet. Exclusion criteria were defined as: suspicion and diagnosis of CVD (angina pectoris, myocardial infarction, stroke, peripheral arterial occlusive disease, heart failure and cardiac arrhythmia), type 1 and 2 diabetes, renal insufficiency and liver diseases, blood coagulation disorders, chronic gastrointestinal disorders (e.g. ulcers, Crohn's disease, pancreatic insufficiency, immunological diseases (e.g. autoimmune diseases)), intake of immunosuppressive drugs or laxatives, intake of supplements containing n-3 PUFA, alcohol, drug and/or medicine dependency, pregnancy or lactation, retraction of the consent by the subject, concurrent participation in another clinical study and participation in a study in the last $30 \mathrm{~d}$. Inclusion and exclusion criteria were assessed using a structured screening questionnaire. Exercise capacity was determined during a resting and exercise electrocardiogram, implemented by trained professionals and a physician. This study was conducted according to the guidelines in the Declaration of Helsinki, and all procedures involving human subjects were approved by the Ethics Commission of the Medical Chamber of Lower Saxony (Hannover, Germany) (Bo/07/2018, URL: https://www.drks.de/drks_web/setLocale_ EN.do). This study is registered in the German Clinical Trial Register (DRKS00014322).

The participants were randomly assigned by an independent researcher using stratified randomisation according to the covariates (in descending order: sex, BMI and age) to one of four study groups: (1) control group (CON), (2) exercise only group (EX), (3) exercise and dietary counseling group (EXDC) and (4) exercise and CO supplementation group (EXCO). However, the present work focuses on the EX and EXCO groups only.

Both groups were instructed to perform exercise training twice a week and maintain their habitual diet. The exercise training was performed in fitness centres and consisted of a warm up followed by two passes of a strength-endurance circuit. The strength training consisted of six machine supported exercises that included all major muscle groups and were performed for $1 \mathrm{~min}$ each. During the initial training session, a maximum force test with three tries was performed. The best of the three tries was scored and used to set the machines to $60 \%$ of the participants' maximum force for the first 2 weeks of training. For the subsequent 6 weeks, the load was increased by $10 \%$ and again by $5 \%$ for the last 4 weeks. The endurance exercise consisted of a 4-min bout performed on bicycle ergometers and cross-trainers at a perceived exertion that equaled a value of 15 on the Borg-Scale. In between each exercise, the participants had $30 \mathrm{~s}$ of rest. Including the warm-up and rest periods, the training session could be completed in approximately 1 hour. Compliance of the participants was assessed via a training log and a questionnaire at the end of the study.

In addition to the exercise training, participants from the EXCO group received capsules providing $2.0 \mathrm{~g}$ of oil from Calanus finmarchicus (Calanus AS) and were instructed to take them daily. The lipid profile of the capsules is shown in Table 1. More than $80 \%$ of the fatty acids are bound as wax esters. The tolerability of the CO capsules was checked using questionnaires.

Compliance of participants and adherence to the instructions were monitored via fortnightly phone calls. Additionally, participants from the EXCO were instructed to return leftover capsules, which were then counted by study personal after delivery. Participants had to consume at least $90 \%$ of the capsules to be considered as compliant.

Table 1. Fatty acid composition of the Calanus finmarchicus oil (CO) used in the study

\begin{tabular}{llcc}
\hline & & $\mathrm{mg} / 100 \mathrm{~g}$ & $\mathrm{mg} / 2 \mathrm{~g}$ \\
Fatty acid* & Common name & $\mathrm{CO}$ & \multicolumn{1}{c}{ CO } \\
\hline $14: 0$ & Myristic acid & 6232 & 125 \\
$15: 0$ & Pentadeclic acid & 323 & $6 \cdot 5$ \\
$16: 0$ & Palmitic acid & 5243 & 105 \\
$16: 3$ & & 325 & 7 \\
$18: 0$ & Stearic acid & 399 & 8 \\
$18: 1 n-9$ & Oleic acid & 1783 & 36 \\
$18: 2 n-6$ & Linoleic acid & 552 & 11 \\
$18: 3 n-3$ & $\alpha$-Linolenic acid & 1149 & 23 \\
$18: 3 n-6$ & $\beta$-Linolenic acid & 140 & 3 \\
$18: 4 n-3$ & Stearidonic acid & 6186 & 124 \\
$20: 1 n-9$ & Gondoic acid & 2148 & 43 \\
$20: 4 n-6$ & Arachidonic acid & 169 & 3 \\
$20: 5 n-3$ & EPA & 5439 & 109 \\
$22: 1 n-11$ & Cetoleic acid & 3495 & 70 \\
$22: 5 n-3$ & Docosapentaenoic acid & 254 & 8 \\
$22: 6 n-3$ & DHA & 4342 & 87 \\
$24: 1 n-9$ & & 414 & 8 \\
\hline
\end{tabular}

${ }^{*}>80 \%$ of fatty acids are present as wax esters. 


\section{Dietary intake from background diet}

Dietary intake of the participants was monitored via 3-d dietary food logs at the beginning, after 6 weeks and at the end of the intervention. The records were checked by nutritionists for completeness, readability and plausibility. If necessary, ambiguities were clarified with the participants. Energy and nutrient intake were estimated using the software PRODI6.4® (NutriScience GmbH).

\section{Blood sampling and analysis}

Blood samples were drawn from the participants after an overnight fast $(\geq 10 \mathrm{~h})$ between 06.00 and 10.00 hours by venepuncture of an arm vein using EDTA tubes (Sarstedt AG \& Co. KG). TAG, LDL-cholesterol and HDL-cholesterol were analysed by a photometric method (Beckman Coulter $\mathrm{GmbH}$ ) by an accredited and certified laboratory (Laborärztliche Arbeitsgemeinschaft für Diagnostik und Rationalisierung e.V.). Total cholesterol and LDL:HDL ratio were calculated from LDL and HDL values.

For analysis of O3I, EDTA tubes were centrifuged for $10 \mathrm{~min}$ at $3000 \mathrm{rpm}$, buffy coat was removed and erythrocytes frozen at $-80^{\circ} \mathrm{C}$ till analysis. The analysis was performed by the Omegametrix laboratory (Martinsried) according to the HS- $n-3$ Index methodology ${ }^{(10,12)}$ by GC. Accordingly, methyl esters of fatty acids were generated from erythrocytes by acid transesterification, and analysis performed using a GC2010 gas chromatograph (Shimadzu) equipped with a SP2560, $100 \mathrm{~m}$ column (Supelco). Hydrogen was used as the carrier gas. To identify fatty acids, a standard mixture characteristic for erythrocytes was used. The results obtained are given as percentage of total identified fatty acids after response factor correction. The O3I represents the sum of EPA + DHA in relation to total fatty acid content in red blood cell membranes. Quality was assured according to DIN ISO 15189.

\section{Statistical analyses}

Data for this analysis were derived from a larger interventional trial, which served as an explorative study. Based on an $\alpha$ of 0.05 and $0 \cdot 80 \beta$, assuming an effect size of more than $0 \cdot 8$, a sample size of $n 25$ was needed to detect between-group differences. Estimating a dropout rate of $15 \%$ at least thirty participants per intervention group were recruited. For this analysis, a power calculation was performed using an online calculator ${ }^{(15)}$. As CO was demonstrated to be equally bioavailable as ethyl esters ${ }^{(9)}$, the calculation was based on a study by Köhler et al. ${ }^{(16)}$. In the study by Köhler et al. ${ }^{(16)}$, participants received $250 \mathrm{mg}$ ethyl-ester bound $\mathrm{EPA}+\mathrm{DHA}$ in the form of enriched sausages which resulted in an O3I increase from 4.18 (SD 0.54) to 5.72 (SD 0.66) \% after 8 weeks. Therefore, we assumed that a change of the O3I of at least $1 \%$ could be achieved after 12 weeks of CO supplementation providing about $200 \mathrm{mg}$ EPA + DHA. Using the online calculator with alpha set to 0.05 and $n 50$ total participants, assuming a mean difference of 1.0 and a $0.7 \%$ standard deviation (using 5.72 (SD 0.66) \% from Köhler et al. ${ }^{(16)}$ as the upper level for the standard deviation) a $99 \%$ probability to detect a difference if the true difference between groups is $1 \%$ was calculated.
Statistical analyses were performed for both the primary outcome O3I and the secondary outcomes (anthropometric data, dietary intake and other fatty acids in erythrocytes). Data are presented as mean values and standard deviations. Distribution of all data was assessed using the Shapiro-Wilk test and Gaussian distribution. Based on the distribution of data, differences in baseline characteristics were assessed using the Mann-Whitney $U$ test or unpaired $t$ test for continuous variables and the $\chi^{2}$ test for nominal variables. Further analysis was performed with data obtained from all participants that arrived at the first and second day of examination $\left(\mathrm{n}_{\mathrm{EX}} 6, \mathrm{n}_{\mathrm{EXCO}} 3\right)$. For analysis, not normally distributed data were log transformed, except C18:2n-6tt which was square root transformed, and differences were assessed using two-factor repeated measures ANOVA using time $\left(t_{0}\right.$ and $\left.t_{12}\right)$ intervention (EX and EXCO). If statistically significant differences between groups were detected, a post hoc analysis with Bonferroni correction was performed within both groups. $P$ values of $<0.05$ were considered as significant. All statistical analyses were carried out using SPSS software (version 23.0; SPSS Inc.).

\section{Results}

\section{Baseline characteristics}

Data analysed were obtained from a total of seventy-two participants ( $68 \%$ female, $32 \%$ male). Due to health and personal reasons, the study had a dropout of nine subjects $\left(n_{\mathrm{EX}} 6, n_{\mathrm{ExCO}} 3\right)$. Baseline characteristics of the study population are shown in Table 2. The mean age was 59.2 (SD 6.2) years, and the average BMI was 27.7 (SD 5.28$) \mathrm{kg} / \mathrm{m}^{2}$. There were no differences in anthropometric parameters or blood lipids between both groups. The study population was slightly hypercholerosterolaemic.

Table 2. Baseline characteristics of all participants* (Mean values and standard deviations)

\begin{tabular}{|c|c|c|c|c|c|}
\hline \multirow[b]{2}{*}{ Measure } & \multicolumn{2}{|c|}{$\begin{array}{l}\text { Exercise } \\
(n 36)\end{array}$} & \multicolumn{2}{|c|}{$\begin{array}{c}\text { Exercise }+\mathrm{CO} \\
(n 36)\end{array}$} & \multirow{2}{*}{$\begin{array}{c}\text { Group } \\
\text { difference: } P\end{array}$} \\
\hline & Mean & SD & Mean & SD & \\
\hline $\operatorname{Sex}(f / m)$ & $24 / 12$ & - & $25 / 11$ & - & 0.80 \\
\hline Age (years) & $59 \cdot 70$ & $6 \cdot 39$ & $58 \cdot 5$ & $5 \cdot 69$ & 0.51 \\
\hline Height $(\mathrm{m})$ & $170 \cdot 9$ & $8 \cdot 73$ & $171 \cdot 3$ & 8.74 & 0.89 \\
\hline Body weight $(\mathrm{kg})$ & 81.9 & $18 \cdot 6$ & $80 \cdot 7$ & $20 \cdot 3$ & 0.61 \\
\hline BMI $\left(\mathrm{kg} / \mathrm{m}^{2}\right)$ & 28.00 & $5 \cdot 51$ & $27 \cdot 28$ & $5 \cdot 26$ & 0.61 \\
\hline $\mathrm{SBP}(\mathrm{mmHg})$ & $129 \cdot 4$ & $14 \cdot 7$ & $134 \cdot 1$ & $20 \cdot 1$ & 0.27 \\
\hline $\mathrm{DBP}(\mathrm{mmHg})$ & $76 \cdot 2$ & $6 \cdot 16$ & 78.6 & 7.91 & 0.15 \\
\hline Pulse (bpm) & $74 \cdot 6$ & 9.58 & $73 \cdot 8$ & $8 \cdot 25$ & 0.71 \\
\hline $\begin{array}{l}\text { Total cholesterol } \\
(\mathrm{mmol} / \mathrm{l})\end{array}$ & $6 \cdot 15$ & 1.01 & $6 \cdot 26$ & 0.96 & 0.97 \\
\hline $\begin{array}{l}\text { HDL-cholesterol } \\
\text { (mmol/l) }\end{array}$ & 1.75 & 0.40 & $1 \cdot 76$ & 0.47 & 0.76 \\
\hline $\begin{array}{l}\text { LDL-cholesterol } \\
\qquad(\mathrm{mmol} / \mathrm{l})\end{array}$ & $3 \cdot 89$ & 0.82 & 3.93 & 0.76 & 0.83 \\
\hline TAG (mmol/l) & 1.31 & 0.63 & $1 \cdot 23$ & 0.39 & 0.56 \\
\hline
\end{tabular}

$\mathrm{CO}$, Calanus finmarchicus oil; f, female; m, male; SBP, systolic blood pressure; DBP, diastolic blood pressure.

* Distribution of sexes between groups was analysed using the $\chi^{2}$ test. All other group differences were assessed with the Mann-Whitney $U$ test or unpaired $t$ test. 


\section{Dietary intake from background diet}

The intake of PUFA and especially EPA and DHA showed high variability at both time points (Table 3 ). Throughout the study, the intake of individual and total fatty acids did not change significantly. Additionally, all other dietary variables did also not change significantly. However, there was a non-significant trend to a lower energetic intake of about $837 \mathrm{~kJ}(200 \mathrm{kcal})$ at the end of the intervention in both study groups.

\section{Tolerability of the Calanus finmarchicus oil capsules}

At the beginning of the study, one participant reported suffering from diarrhoea after taking the CO capsules. Despite the instructions to take the capsules with food, this participant took the capsules on an empty stomach. After starting taking the capsules as suggested, the symptoms disappeared. Otherwise, no adverse symptoms after the CO capsule intake were reported.

\section{Fatty acid content of erythrocytes and omega-3 index}

Comparison of baseline values between the two study groups showed no significant differences in fatty acid levels. In both groups, PUFA values declined in the following order ARA $>$ $\mathrm{LA}>\mathrm{DHA}>\mathrm{DPA} n-3=\mathrm{C} 22: 4 n-6>\mathrm{C} 20: 3 n-6>\mathrm{EPA}>\mathrm{DPA} n-6>$ ALA $=\mathrm{C} 20: 2 n-6>\mathrm{C} 18: 3 n-6$. Lowest quantities were observed for SDA. O3I was 6.01 (SD 1.26) \% in EX and 6.07 (SD 1.29) \% in EXCO (Table 4).

During the study, a significant increase in $\mathrm{O} 3 \mathrm{I}$ occurred in the EXCO group from 6.07 (sD 1.29) to 7.37 (sD 1.10$) \%(P<0.001)$. In line, EPA increased from 0.92 (SD 0.42 ) to 1.32 (SD 0.37) \% $(P<0.001)$ and DHA from 5.15 (sD 1.03) to 6.04 (SD 0.94) changes $(\Delta)^{*}$

(Mean values and standard deviations)
$(P<0 \cdot 001)$. Further, relevant increases occurred in SDA from 0.027 (sD 0.008$)$ to 0.038 (sD 0.012$) \%(P=0.008)$ and DPA $n-3$ from 2.67 (sD 0.41$)$ to 2.86 (sD 0.41$) \%(P<0.001)$. In contrast, levels of most $n$-6-PUFA slightly decreased in consequence of the intervention: ARA decreased from 15.3 (SD 1.07) to 14.9 (sD 0.94$) \%(P<0.001), \mathrm{C} 22: 4 n-6$ from 2.70 (SD 0.48$)$ to 2.46 (sD 0.42$) \%(P<0.001)$ and DPA $n-6$ from 0.63 (sD 0.18$)$ to 0.56 (sD $0 \cdot 18) \%(P<0 \cdot 001)$.

The EX group showed no physiologically relevant changes in erythrocyte fatty acid levels throughout the intervention.

\section{Discussion}

Current evidence from preclinical studies indicates promising effects of CO on obesity and obesity-related inflammation as well as on blood glucose control and atherosclerosis ${ }^{(17-19)}$. However, the underlying physiological and molecular mechanisms of action are not yet fully understood. An important prerequisite when examining the physiological effects of marine oils is to ensure that active ingredients enter the body in sufficiently high amounts $^{(11)}$. CO contains the $n-3$ PUFA SDA, EPA and DHA. Hence, the investigation of the effect of $n-3$ PUFA from CO on PUFA blood levels is necessary to understand the mode of action of this novel marine oil.

In evaluating the effect of fatty acids, several factors have to be considered with one of the first being the chemical binding form ${ }^{(11)}$. Commercially available marine $n$-3 PUFA supplements contain fatty acids in the form of TAG, ethyl esters or phospholipids. Contrary to that, $>80 \%$ of fatty acids in CO are bound to fatty alcohols, which classifies them as wax esters.

Table 3. Dietary energy and nutrient intake calculated from 3-d dietary records at the beginning (0) and at the end (12) of the intervention as well as absolute

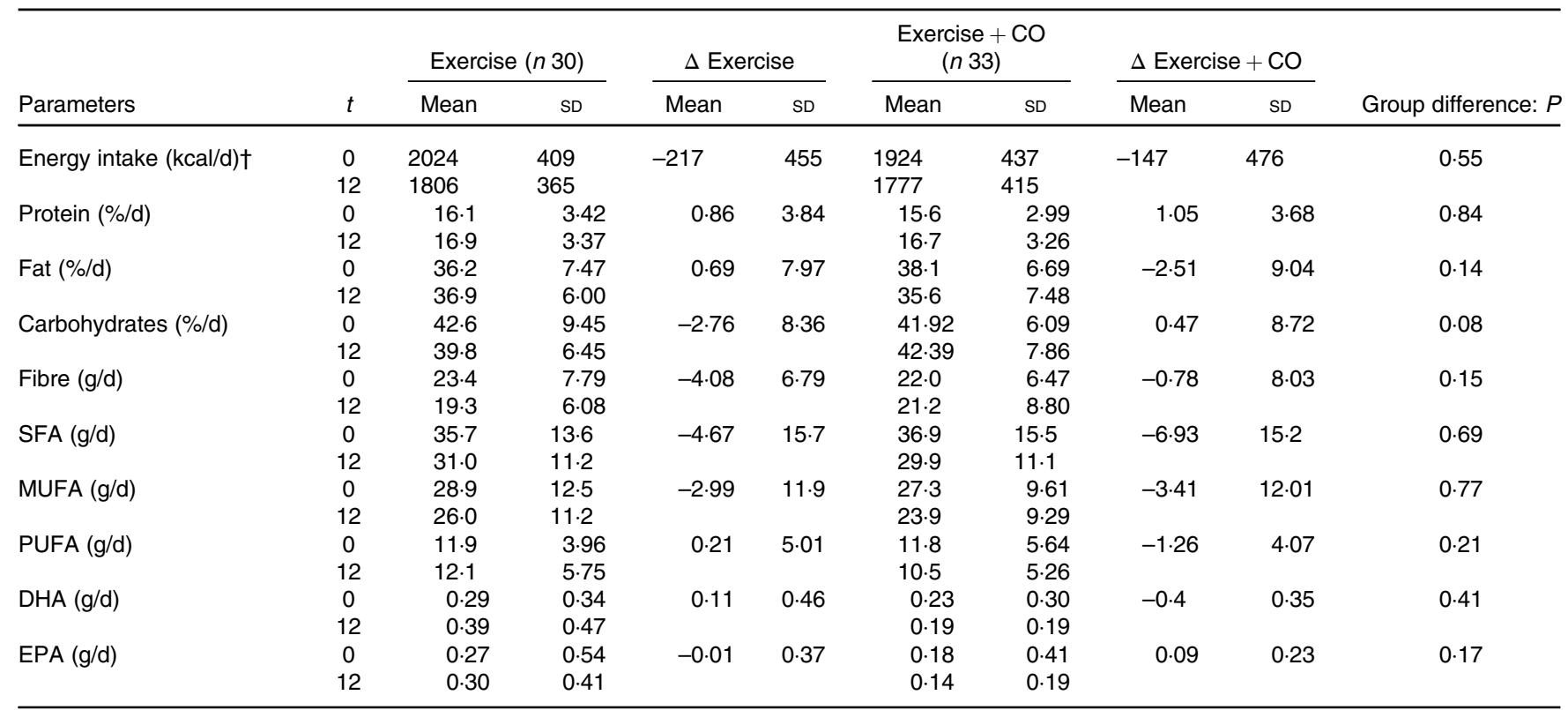

CO, Calanus finmarchicus oil; $t$, times in weeks.

${ }^{*} P$ values represent time $\times$ intervention interaction analysed with two-way repeated-measures ANOVA.

† To convert kcal to kJ, multiply by $4 \cdot 184$. 
Table 4. Fatty acid content of erythrocytes (given as percentage of total fatty acids) at the beginning (0) and at the end (12) of the study and absolute changes study $(\Delta)^{*}$

(Mean values and standard deviations)

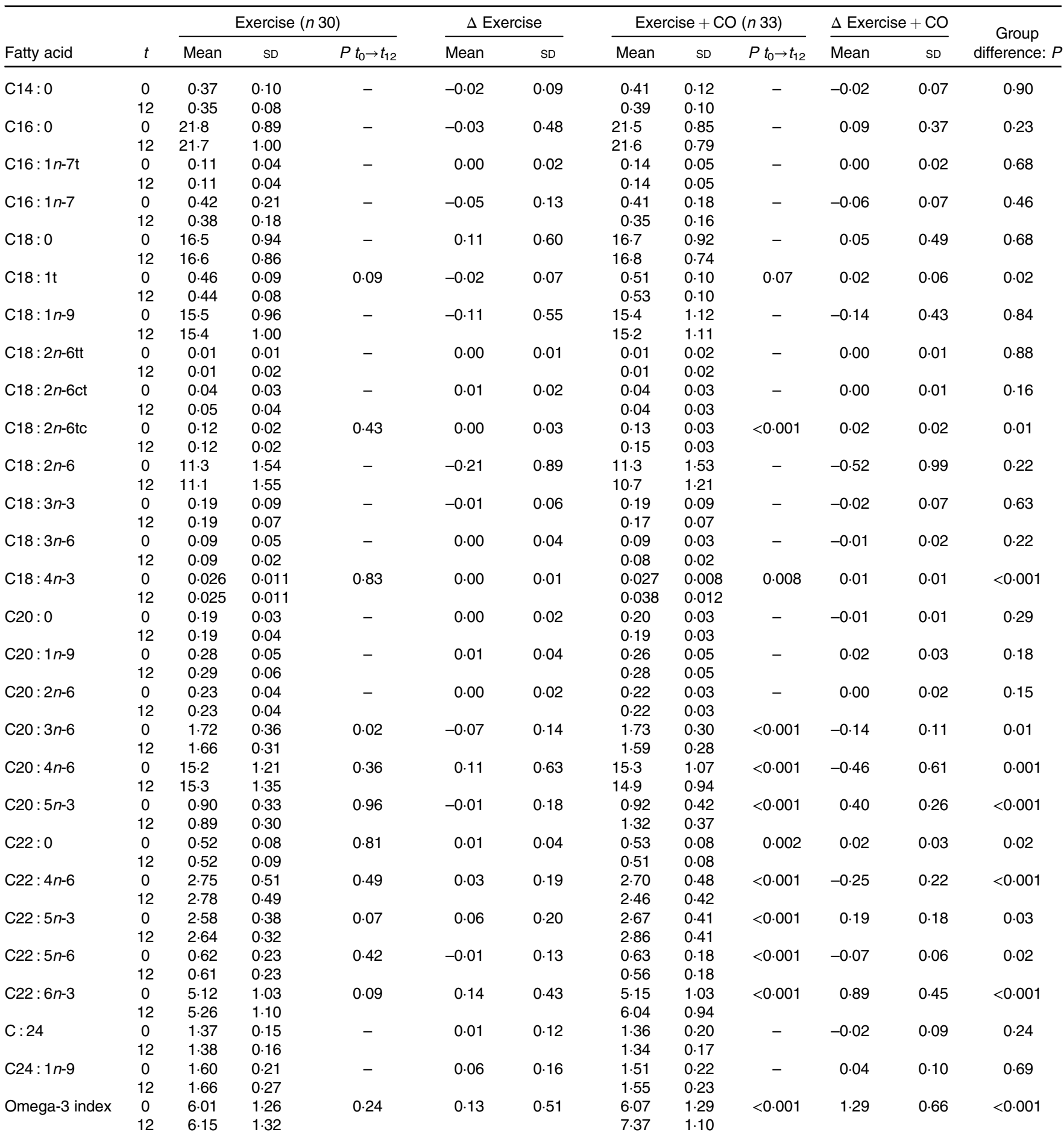

CO, Calanus finmarchicus oil; $t$, times in weeks.

${ }^{*} P$ values represent time $\times$ intervention interaction analysed with two-way repeated-measures ANOVA. In the case of significance, statistical differences within groups were detected with Bonferroni's post hoc test.

Overall wax esters have been discussed as both poorly digestible and bioavailable in mammals ${ }^{(20)}$ and larger amounts are reported to cause gastrointestinal symptoms in humans ${ }^{(21)}$. In the present study, $\mathrm{CO}$ was well tolerated. Only one case reported gastrointestinal discomfort (diarrhoea) after ingesting the CO capsules on an empty stomach in the first week of intervention. Those symptoms disappeared immediately after the participant started following the instructions to take the 
capsules together with a meal. With regard to the bioavailability of wax esters, a single-dose study conducted by Cook et al. demonstrated that the bioavailability of wax esters from CO is comparable with ethyl esters from fish oil ${ }^{(9)}$. In the mentioned study, the bioavailability of $260 \mathrm{mg}$ EPA and $156 \mathrm{mg}$ DHA from $4 \mathrm{~g}$ of CO $v .465 \mathrm{mg}$ EPA and $375 \mathrm{mg}$ DHA from $1 \mathrm{~g}$ of ethyl esters from fish oil were compared over a $72 \mathrm{~h}$. Although the dose of $\mathrm{EPA}+\mathrm{DHA}$ from fish oil was almost twice as high as from CO (840 v. $416 \mathrm{mg}$ ), no significant differences in bioavailability were found. Moreover, plasma levels of EPA remained even higher in the CO group 24-72 h after intake, indicating that wax esters may be a highly available EPA/DHA source. However, the effect of a longer-term CO intake on EPA and DHA blood levels has not been studied.

In the present study, a 12 -week intake of $2 \cdot 0 \mathrm{~g} \mathrm{CO}$, providing about $200 \mathrm{mg}$ of EPA + DHA, led to a significant increase of the O3I from 6.07 (SD 1.29) to 7.37 (SD 1.10) \%, which is an increase of 1.29 (sD 0.66 ) \%. In contrast, no difference was found in the control group that performed exercise only, indicating that engagement in a moderate exercise programme does not negatively influence the O3I.

With regard to the low EPA + DHA dose used in this study, there are only four studies using comparable doses ${ }^{(16,22-24)}$. Köhler et $a l{ }^{(16)}$ investigated the effect of DHA + EPA from enriched sausages containing $250 \mathrm{mg} / \mathrm{d}$ EPA + DHA bound as ethyl esters and $250 \mathrm{mg} / \mathrm{d}$ ALA bound as TAG. A control group received sausages containing only $250 \mathrm{mg} / \mathrm{d}$ of ALA. After a study period of 8 weeks, a $1.5 \%$ increase in O3I from 4.18 (sD 0.54 ) to 5.72 (sD 0.66) \% was reported for the group consuming the enriched sausages, while no difference was observed for the control group. Another study reported significant improvements of the O3I after supplementing different doses of $\mathrm{EPA}+\mathrm{DHA}$ using krill oil ${ }^{(24)}$. In krill oil, EPA + DHA are mainly bound in phospholipids. Two hundred and $400 \mathrm{mg} / \mathrm{d}$ $\mathrm{EPA}+\mathrm{DHA}$, respectively, led to an O3I increase from 3.56 (SD 0.82 ) to 4.19 (SD 0.79 ) \% and 4.0 (SD 0.88 ) to 5.17 (SD 0.96$) \%$, respectively ${ }^{(24)}$. Flock et al. $^{(23)}$ reported an 1.88 (sD 0.23$) \%$ O3I increase (4.29 (sD 0.22) to 6.19 (SD 0.23)\%) after a 20 -week supplementation of about $300 \mathrm{mg} / \mathrm{d}$ TAG-bound $\mathrm{EPA}+\mathrm{DHA}$, while Sarter et al. reported an increase of $1.7 \%$ (3.9 (sD 1.0) to 4.8 (SD 0.8) \%) after 16-week intake of $254 \mathrm{mg} / \mathrm{d}$ TAG-bound EPA $+\mathrm{DHA}^{(22)}$. Compared with the four previous studies, participants from this study showed an overall better $\mathrm{EPA}+$ DHA supply status with baseline values of about $6 \%$. This is of interest because it has been demonstrated that the response of O3I to $n$-3 PUFA supplementation is dependent on the baseline O3I, with lower O3I leading to greater O3I responses $^{(25,26)}$. Nonetheless, the low dose of EPA + DHA from CO still successfully improved the O3I to an extent close to improvements observed in comparable studies with populations that had substantially lower baseline O3I values. Noteworthy, increases of only $1 \%$ were reported to already decrease the risk for sudden cardiac death ${ }^{(27)}$.

However, the effect of CO supplementation on the O3I is unlikely to be solely due to the gastrointestinal uptake of preformed EPA and DHA. It is more likely that SDA also contributes to the O3I increase as SDA is the most abundant $n-3$ PUFA in CO and one of the precursors in the metabolic pathway of EPA and
DHA synthesis. When compared among each other, $2 \cdot 0 \mathrm{~g}$ of CO provided $124 \mathrm{mg}$ SDA but only $109 \mathrm{mg}$ EPA and $87 \mathrm{mg}$ DHA. In a recent study, we investigated the short-term effect of a single-dose intake of $26 \mathrm{~g}$ echium oil containing $3 \mathrm{~g}$ of SDA and reported significant increases of EPA (47\%) and DHA (21\%) levels in plasma after $72 \mathrm{~h}^{(28)}$. Moreover, in a previous long-term study on SDA and O3I, a 16-week supplementation of SDA enriched soyabean oil providing $3.66 \mathrm{~g}$ SDA led to a $19.5 \%$ increase of the $\mathrm{O} 3 \mathrm{I}^{(29)}$. However, no significant changes in DHA were observed, while EPA and SDA levels increased in erythrocytes. In a recent study, we observed a similar outcome after a 12-week supplementation of $12.9 \mathrm{~g} \mathrm{ALA}^{(30)}$. In comparison, the 12 -week intake of the low $n$ - 3 PUFA dose in the present study led to increases of $41 \%$ in SDA, $44 \%$ in EPA and $17 \%$ in DHA. Noteworthy, CO also contains a non-negligible amount (about $70 \mathrm{mg} / 2 \mathrm{~g} \mathrm{CO}$ ) of $22: 1 n$ - 11 (cetoleic acid). A recent study demonstrated that cetoleic acid stimulated the conversion of ALA to EPA and DHA in human hepatocytes ${ }^{(31)}$. Therefore, it can be hypothesised that this fatty acid could have contributed to the observed elevated EPA + DHA levels in erythrocytes.

Furthermore, it is important to note that the participants of the present study can be classified as pre-obese according to the mean BMI. This is of importance as obesity is known to affect lipid metabolism ${ }^{(32,33)}$. The slightly elevated cholesterol levels in our study collective also reflect a pre-obese state. Moreover, body weight has been found to be a modulator of the response to supplementation of EPA + DHA where higher body weight is associated with a weaker response to a given amount of $\mathrm{EPA}+\mathrm{DHA}^{(23)}$. Beyond this background, the present results are of interest as the low dose of EPA + DHA from CO significantly increased the $\mathrm{O} 3 \mathrm{I}$ in this pre-obese study collective.

Finally, to fully elucidate the impact of CO supplementation on fatty acid metabolism and EPA + DHA status, further bioavailability studies are needed. Preferably, this should also be combined with a strictly controlled diet and PUFA intake.

\section{Limitations}

As data for this analysis were obtained from a larger interventional trial, there are also some methodical limitations. As mentioned above, when evaluating PUFA uptake, it is preferable to strictly control for dietary intake. That being said, one limitation of our study was the estimation of the EPA and DHA intake via 3-d food logs, as this data are self-reported and prone to over- or underestimation as well as different food selection of the participants. For example, if a participant eats a fish meal on $1 \mathrm{~d}$ during the 3-d food logs, this leads to massive fluctuations in the mean $\mathrm{EPA}+\mathrm{DHA}$ intake levels of the background diet in each group. Moreover, EPA + DHA composition estimated from food logs should be evaluated cautiously as it may be incorrect due to errors in EPA + DHA compositions found in the food databases from nutrient intake calculation software ${ }^{(34)}$. This is also reflected by the variability of the estimated dietary PUFA intake levels at the beginning and at the end of the study. However, as no significant changes in dietary EPA and DHA intake from baseline until after the intervention could be detected among the study groups and, more importantly, no changes in erythrocyte PUFA levels in the EX group were seen, EPA and DHA intake 
from the background diet can be excluded as a potential confounder in this study. Another limitation of this study is the lack of a placebo group due to the original study design, which encompassed four study groups and did not account for a placebo. Future studies should be conducted with a placebo group.

\section{Conclusion}

This is the first study to show that intake of $2 \mathrm{~g}$ CO over a period of 12 weeks significantly improves the O3I in elderly participants engaging in a moderate exercise intervention while exercise alone did not affect the O3I. These data provide the first indication that wax ester-bound $n-3$ PUFA from CO are well absorbed and are suited to cover the $n$-3 PUFA supply. Future studies should investigate the long-term bioavailability of $n$-3 PUFA from CO compared with TAG- or ethyl ester-bound $n-3$ PUFA from fish oil or phospholipid-bound $n-3$ PUFA from krill oil.

\section{Acknowledgements}

First of all, the authors would like to thank all participants who took part in our study, and the fitness centres that supported the implementation of this study. Further, the authors thank Calanus AS for providing the CO capsules, Heike Kohrs for technical assistance and Dr Erinn Gideons for proofreading our manuscript.

This research received no external financial support.

All authors have read and agreed to the published version of the manuscript. P. W., T. K. B., K. K. and A. H. formulated the research question and designed the study. P. W. and J. N. carried out the study. P. W. analysed the data. P. W., J. P. S. and A. H. interpreted the findings and P. W. and J. P. S. wrote the article.

The authors declare no conflict of interest. The CO capsules were provided by Calanus AS, Norway. The company had no role in the design of the study; in the collection, analyses or interpretation of data; in the writing of the manuscript or in the decision to publish the results.

\section{References}

1. Ruxton CHS, Reed SC, Simpson MJA, et al. (2004) The health benefits of omega-3 polyunsaturated fatty acids: a review of the evidence. J Hum Nutr Diet 17, 449-459.

2. Calder PC \& Yaqoob P (2009) Omega-3 polyunsaturated fatty acids and human health outcomes. Biofactors 35, 266-272.

3. International Society for the Study of Fatty Acids and Lipids (2004) Report of the Sub-Committee on Recommendations for intake of polyunsaturated fatty acids in healthy adults. https://www.issfal.org/assets/issfal\%2003\%20pufaintakereccomd finalreport.pdf (accessed August 2020).

4. Micha R, Khatibzadeh S, Shi P, et al. (2014) Global, regional, and national consumption levels of dietary fats and oils in 1990 and 2010: a systematic analysis including 266 countryspecific nutrition surveys. BMJ 348, g2272.

5. Stehle P (2014) The Nutrition Report 2012 summary. EJNFS $\mathbf{4}$, $14-62$.

6. Lee JH, O'Keefe JH, Lavie CJ, et al. (2009) Omega-3 fatty acids: cardiovascular benefits, sources and sustainability. Nat Rev Cardiol 6, 753-758.
7. Pedersen AM, Vang B \& Olsen RL (2014) Oil from Calanus finmarchicus - composition and possible use: a review. J Aquat Food Prod Technol 23, 633-646.

8. Calder PC (2015) Marine omega-3 fatty acids and inflammatory processes: effects, mechanisms and clinical relevance. Biochim Biophys Acta Mol Cell Biol Lipids 1851, 469-484.

9. Cook CM, Larsen TS, Derrig LD, et al. (2016) Wax ester rich oil from the Marine Crustacean, Calanus finmarchicus, is a bioavailable source of EPA and DHA for human consumption. Lipids 51, 1137-1144.

10. Harris WS (2008) The omega-3 index as a risk factor for coronary heart disease. Am J Clin Nutr 87, 1997S-2002S.

11. Schuchardt JP \& Hahn A (2013) Bioavailability of long-chain omega-3 fatty acids. Prostaglandins Leukot Essent Fatty Acids 89, 1-8.

12. von Schacky C (2014) Omega-3 index and cardiovascular health. Nutrients 6, 799-814.

13. von Schacky C, Kemper M, Haslbauer R, et al. (2014) Low omega-3 index in 106 German elite winter endurance athletes: a pilot study. Int J Sport Nutr Exerc Metab 24, 559-564.

14. Wilson PB \& Madrigal LA (2016) Associations between whole blood and dietary omega-3 polyunsaturated fatty acid levels in collegiate athletes. Int J Sport Nutr Exerc Metab 26, 497-505.

15. Schoenefeld DA (2015) Statistical considerations for clinical trials and scientific experiments. http://hedwig.mgh.harvard. edu/sample_size/size.html (accessed May 2019).

16. Köhler A, Heinrich J \& von Schacky C (2017) Bioavailability of dietary omega-3 fatty acids added to a variety of sausages in healthy individuals. Nutrients $\mathbf{9}, 629$.

17. Höper AC, Salma W, Khalid AM, et al. (2013) Oil from the marine zooplankton Calanus finmarchicus improves the cardiometabolic phenotype of diet-induced obese mice. BrJ Nutr 110, 2186-2193.

18. Höper AC, Salma W, Sollie SJ, et al. (2014) Wax esters from the marine copepod Calanus finmarchicus reduce diet-induced obesity and obesity-related metabolic disorders in mice. J Nutr 144, 164-169.

19. Eilertsen K-E, Mæhre HK, Jensen IJ, et al. (2012) A wax ester and astaxanthin-rich extract from the marine copepod Calanus finmarchicus attenuates atherogenesis in female apolipoprotein E - deficient mice. J Nutr 142, 508-512.

20. Place AR (1992) Comparative aspects of lipid digestion and absorption: physiological correlates of wax ester digestion. Am J Physiol 263, R464-R471.

21. Ho Ling K, Nichols PD \& But PP (2009) Fish-induced keriorrhea. Adv Food Nutr Res 57, 1-52.

22. Sarter B, Kelsey KS, Schwartz TA, et al. (2015) Blood docosahexaenoic acid and eicosapentaenoic acid in vegans: associations with age and gender and effects of an algal-derived omega-3 fatty acid supplement. Clin Nutr 34, 212-218.

23. Flock MR, Skulas-Ray AC, Harris WS, et al. (2013) Determinants of erythrocyte omega-3 fatty acid content in response to fish oil supplementation: a dose-response randomized controlled trial. J Am Heart Assoc 2, e000513.

24. Berge K, Musa-Veloso K, Harwood M, et al. (2014) Krill oil supplementation lowers serum triglycerides without increasing low-density lipoprotein cholesterol in adults with borderline high or high triglyceride levels. Nutr Res 34, 126-133.

25. Cao J, Schwichtenberg KA, Hanson NQ, et al. (2006) Incorporation and clearance of omega-3 fatty acids in erythrocyte membranes and plasma phospholipids. Clin Chem 52, $2265-2272$.

26. Keenan AH, Pedersen TL, Fillaus K, et al. (2012) Basal omega-3 fatty acid status affects fatty acid and oxylipin responses to high-dose $n$-3-HUFA in healthy volunteers. J Lipid Res 53, 1662-1669. 
27. Harris WS \& von Schacky C (2008) Omega-3 fatty acids, acute coronary syndrome, and sudden death. Curr Cardio Risk Rep 2, 161-166.

28. Greupner T, Koch E, Kutzner L, et al. (2019) Single-dose SDA-rich echium oil increases plasma EPA, DPA $n-3$, and DHA concentrations. Nutrients 11, 2346.

29. Harris WS, Lemke SL, Hansen SN, et al. (2008) Stearidonic acid-enriched soybean oil increased the omega-3 index, an emerging cardiovascular risk marker. Lipids 43, 805-811.

30. Greupner T, Kutzner L, Nolte F, et al. (2018) Effects of a 12-week high- $\alpha$-linolenic acid intervention on EPA and DHA concentrations in red blood cells and plasma oxylipin pattern in subjects with a low EPA and DHA status. Food Funct 9, 1587-1600.
31. Østbye T-KK, Berge GM, Nilsson A, et al. (2019) The long-chain monounsaturated cetoleic acid improves the efficiency of the $n$-3 fatty acid metabolic pathway in Atlantic salmon and human HepG2 cells. Br J Nutr 122, 755-768.

32. Singla P (2010) Metabolic effects of obesity: a review. World J Diabetes 1, 76-88.

33. Bays HE, Toth PP, Kris-Etherton PM, et al. (2013) Obesity, adiposity, and dyslipidemia: a consensus statement from the National Lipid Association. J Clin Lipidol 7, 304-383.

34. Archer E, Hand GA \& Blair SN (2013) Validity of U.S. Nutritional Surveillance: National Health and Nutrition Examination Survey Caloric Energy Intake Data, 1971-2010. PLOS ONE 8, e76632. 